\title{
Sobre el derecho humano al agua
}

WILliam POSTIGO DE LA MOTTA

Ex Adjunto al Defensor del Pueblo de Perú para los Servicios Públicos y Medio Ambiente.

\section{RESUMEN}

Con base en los acuerdos internacionales y la Constitución peruana, este ensayo sostiene que el marco legal en Perú reconoce el derecho al agua potable. Se analizan las causas de por qué este derecho no es vigente en la realidad, particularmente con referencia a los problemas de gestión y económicos de las empresas estatales. Asimismo, se discuten los conceptos de bien público y bien de dominio público como marco para analizar el supuesto conflicto entre el reconocimiento de un derecho humano al agua y el valor económico de este recurso. El autor plantea que la participación privada para proveer capacidad de gestión y capital se justifica por la escasez de ambos en el sector público. Sin embargo, se analizan también los riesgos de la intervención privada, la cual es considerada como complementaria antes que opuesta a la gestión pública.

Palabras clave: derechos humanos, agua potable, servicios públicos, gestión pública, privatización.

\section{INTRODUCCIÓN}

Millones de personas en el Perú carecen de acceso a los servicios de agua potable y alcantarillado, no obstante la importancia vital de estos servicios para la calidad de vida de las personas. Precisamente esta importancia ha determinado que el derecho internacional haga un reconocimiento explícito del derecho humano al agua potable para todos. A pesar de ello, y no obstante que el texto constitucional también permite el reconocimiento de un derecho al agua para todos los peruanos, las empresas prestadoras de estos servicios no se encuentran en condiciones de garantizar este derecho. 
El presente ensayo analiza por qué el derecho humano al agua en Perú no es vigente, y las opciones de política existentes para garantizarlo. En la primera sección se analiza el concepto del derecho al agua potable, en el cual se incluye el alcantarillado, para sustentar la obligación del Estado de garantizar el acceso de todos los ciudadanos a estos servicios. En la segunda sección se analiza por qué este derecho actualmente no es vigente para millones de peruanos, enfocado en la situación económica de las empresas prestadoras. Finalmente, en la tercera sección, se analizan las ventajas y problemas de una posible participación del sector privado en la prestación de los servicios de agua y alcantarillado. El presente artículo recoge y amplía las ideas y conceptos incluidos en el Informe N. ${ }^{\circ} 94$ de la Defensoría del Pueblo de Perú (2005), elaborado bajo la dirección del autor cuando era funcionario de dicha entidad.

\section{EL DERECho al agua potable}

La Declaración Universal de los Derechos Humanos ha reconocido que las personas tenemos un derecho natural a un conjunto de libertades esenciales, aunque en muchos países estos derechos aún no son respetados plenamente. Sin embargo, la humanidad ha progresado sostenidamente hacia su reconocimiento universal, lo cual se manifiesta en la incorporación de tales derechos dentro de la legislación de la mayoría de los Estados nacionales. Más recientemente, el derecho internacional ha venido reconociendo otros derechos, los cuales se denominan Derechos Económicos, Sociales y Culturales —DESC-, y que amplían los derechos humanos para incluir el derecho a satisfacer un conjunto de necesidades esenciales como alimentación, vestido, vivienda, educación, salud y agua potable, entre otros; además de reconocer el derecho a la identidad cultural.

El Pacto Internacional de Derechos Económicos, Sociales y Culturales del año 1995, del cual el Perú es parte, reconoce en su artículo $12 .^{\circ}$ párrafo 1 el Derecho a la Vida y a la Dignidad Humana, además de precisar que los Estados Partes reconocen el derecho de toda persona al disfrute del más alto nivel posible de salud física y mental. Estos derechos no serían vigentes si las personas carecieran de un acceso adecuado a los servicios de agua potable y alcantarillado. Cabe destacar, a este respecto, que la Organización Mundial de la Salud ha definido a ésta como un estado de completo bienestar físico, mental 
y social y no solamente la ausencia de infecciones o enfermedades. Por tanto, el acceso a los servicios de agua es fundamental para la vigencia del derecho a la salud.

Por su parte, el Protocolo Adicional a la Convención Americana sobre Derechos Humanos en Materia de Derechos Económicos, Sociales y Culturales, "Protocolo de San Salvador» de 1988, Artículo 11.o inciso 1 , señala que toda persona tiene derecho a vivir en un medio ambiente sano y a contar con servicios públicos básicos, de los cuales el más básico es precisamente el agua. De otro lado, el párrafo $1 .^{\circ} \mathrm{del}$ artículo $11 .^{\circ}$ del Pacto Internacional de Derechos Económicos, Sociales y Culturales contiene una serie de derechos que se desprenden del derecho a un nivel de vida adecuado. El Pacto señala que los Estados Partes reconocen el derecho de toda persona a un nivel de vida adecuado para sí y su familia, lo que incluye alimentación, vestido y vivienda, y una mejora continua de las condiciones de existencia. En este contexto, una vivienda carente de los servicios de agua y saneamiento no podría considerarse adecuada.

Las disposiciones del Pacto Internacional de Derechos Económicos, Sociales y Culturales - DESC - son desarrolladas y precisadas por el Comité de Derechos Económicos, Sociales y Culturales de las Naciones Unidas mediante las denominadas Observaciones Generales. Dicho Comité ha hecho un reconocimiento explícito del derecho humano al agua en la Observación General N. ${ }^{\circ} 15$ de 2002. Allí se reconoce "... el derecho de todos a disponer de agua suficiente, salubre, aceptable, accesible y asequible para el uso personal y doméstico. Un abastecimiento adecuado de agua salubre es necesario para evitar la muerte por deshidratación, para reducir el riesgo de las enfermedades relacionadas con el agua y para satisfacer las necesidades de consumo y cocina, y las necesidades de higiene personal y doméstica».

Otros acuerdos internacionales, en que el Estado peruano es parte, contemplan también el derecho al agua como un derecho humano. Tal es el caso de la Convención sobre la Eliminación de todas las formas de Discriminación contra la Mujer, donde se establece que los Estados Partes asegurarán a las mujeres el derecho a "gozar de condiciones de vida adecuadas, particularmente en las esferas de (...) el abastecimiento de agua" (artículo 14..$^{\circ}$, inciso 2). Igualmente, la Convención sobre los Derechos 
del Niño exige a los Estados Partes luchar contra las enfermedades y la malnutrición mediante "el suministro de alimentos nutritivos adecuados y agua potable salubre» (artículo $24^{\circ}$, inciso 2). En Gleick (1998) y Scanlon et al (2004) se encuentra un análisis comprehensivo sobre los acuerdos internacionales, de los cuales puede desprenderse el reconocimiento de un Derecho Humano al Agua.

En adición a los instrumentos internacionales, la Constitución Política del Perú establece un conjunto de derechos, los cuales no podrían realizarse sin el ejercicio del derecho al agua. Entre otros cabe mencionar el derecho de las personas a la protección de su salud, la del medio familiar y la de la comunidad (Art. 7. ${ }^{\circ}$ ), y el derecho a gozar de un ambiente equilibrado y adecuado al desarrollo de su vida (Art. $2 .^{\circ}$, inciso 22). $\mathrm{Al}$ respecto, es importante destacar que existen sentencias del Tribunal Constitucional, de las cuales se desprende que todos los derechos, sean civiles, políticos, económicos, sociales, o culturales, constituyen obligaciones del Estado, y que por tanto éste debe respetarlos y garantizarlos'.

El contenido del Derecho Humano al Agua, así como las obligaciones genéricas del Estado han sido desarrollados tanto por la Defensoría del Pueblo de Perú (2005), como por la Defensoría del Pueblo de Colombia (2005). Cabe precisar, sin embargo, que el reconocimiento del derecho humano al agua no supone la obligación del Estado de proveer gratuitamente los servicios de agua potable y alcantarillado, los cuales deben ser pagados por los usuarios, como es usual. Sí implica, empero, que el Estado está obligado a garantizar un acceso adecuado a los sectores de la población que por su condición de pobreza no estén en condiciones de pagar parte o la totalidad del costo del servicio en un nivel de consumo básico. En tal sentido, los mecanismos de subsidio en sus diversas formas pueden ser un instrumento para que el Estado cumpla esta obligación.

\section{POR QUÉ EL DERECHO AL AGUA NO ESTÁ VIGENTE EN EL PERÚ}

En el Perú la provisión de los servicios de agua potable y alcantarillado se desenvuelve en un marco institucional relativamente diverso. A nivel urbano, predomina el servicio a través de Empresas Prestadoras Municipales, aproximadamente 54 a nivel nacional. En algunas ciudades pequeñas existen también servicios de agua y desagüe directamente administrados por los municipios. Finalmente, en la ciudad 
capital Lima, el servicio es prestado por SEDAPAL, la única empresa que pertenece al Gobierno central. De otro lado, en el ámbito rural existen también diversas formas institucionales, mayormente de naturaleza asociativa, pero predominan las denominadas Juntas Administradoras de Servicios de Saneamiento --JASS-.

La cobertura de agua potable alcanza al 75\% de la población nacional, $82 \%$ de la población urbana. Esto implica que aproximadamente 7 millones de habitantes carecen de agua potable a nivel nacional, unos 3,5 millones a nivel urbano. En alcantarillado, la cobertura nacional es de apenas $57 \%$, y a nivel urbano $71 \%$. En este caso, más de 5 millones de personas carecen de servicios de desagüe en las ciudades y 11,5 millones a nivel nacional. Durante los últimos 15 años la cobertura de estos servicios ha logrado ampliaciones relativamente modestas $y$, en algunas localidades, se observa un deterioro de la calidad del servicio expresado en un menor promedio de horas del servicio y problemas localizados de calidad.

Las empresas prestadoras del ámbito urbano presentan serios problemas de gestión y se caracterizan por una severa restricción financiera, lo que les impide atender las necesidades de expansión del servicio y de mejora de la calidad. El cuadro siguiente muestra los indicadores de resultados y dos indicadores de gestión para algunas empresas. Se observa que los resultados económicos de las empresas seleccionadas son negativos o muy pobres, lo cual constituye una situación general a nivel nacional.

Cabe señalar que durante los años 90 se ejecutaron muchas obras de agua potable y alcantarillado con financiamiento de FONAVI ${ }^{2}$. En muchas empresas estas obras no han sido incorporadas en su patrimonio contable, por lo que dicho patrimonio estaría subestimado, de manera que en tales casos los indicadores de rentabilidad serían aún peores. Dado que las empresas no reciben recursos del Tesoro Público, su fuente de financiamiento para obras es el endeudamiento $y$, en ausencia de éste, las utilidades que pueda generar la propia empre$\mathrm{sa}^{3}$. La crítica situación financiera por la que atraviesan les impide ser sujetas de crédito, además que se requiere autorización del Ministerio de Economía y Finanzas (MEF) para endeudarse, la cual es muy difícil de obtener. Por tanto, al no generar utilidades, a las empresas les resulta imposible financiar las elevadas inversiones requeridas para ampliar la cobertura y mejorar la calidad del servicio. 


\begin{tabular}{|c|c|c|c|c|c|c|c|c|c|c|c|c|}
\hline \multirow{2}{*}{$\begin{array}{l}\text { Empressas } \\
\text { prestadoras }\end{array}$} & \multicolumn{4}{|c|}{ Rentabilidad sobre patrimonio (\%) } & \multicolumn{4}{|c|}{ Aqua no contrabilizada (\%) } & \multicolumn{4}{|c|}{ Nivel de morosidad (meses) } \\
\hline & 2001 & 2002 & 2003 & 2004 & 2001 & 2002 & 2003 & 2004 & 2001 & 2002 & 2003 & 2004 \\
\hline SEOAPN & 0.9 & 0.6 & 1.0 & 2.7 & 42 & 40 & 40 & 37 & 4,6 & 3.1 & 2,6 & 3,0 \\
\hline SEDAPAR & $-3,6$ & $-7,5$ & $-4,8$ & $-1,5$ & 45 & 48 & 46 & 42 & 6,9 & 7,8 & 6.3 & 7,4 \\
\hline SEDALIB & $-7,9$ & $-5,2$ & $-13,2$ & $-3,4$ & 41 & 45 & 47 & 50 & 10,7 & 8,5 & 8,4 & 8,1 \\
\hline EPS GRAU & $-57,7$ & $-22,8$ & $-12,2$ & 3.6 & 60 & 65 & 63 & 61 & 6,6 & 15,3 & 15,6 & 14,4 \\
\hline SEDACUSCO & 1,1 & $-0,1$ & 0,6 & S.I. & 47 & 50 & 50 & 48 & 2,7 & 1,8 & 1,3 & 1,0 \\
\hline EPSASA & $-6,6$ & $-1,6$ & $-1,7$ & 0,1 & 52 & 53 & 52 & 47 & 1,9 & 1,9 & 1,6 & 1.8 \\
\hline
\end{tabular}

Fuente: Indicadores de Gestión SUNASS.

La otra parte del cuadro muestra los serios problemas de gestión que enfrentan las empresas. Como se aprecia, las pérdidas de agua son mayores al $40 \%$ de lo producido, y en algunos casos superan el $50 \%$. Asimismo, la morosidad, excepto en SEDACUSCO y EPSASA ${ }^{4}$ (Ayacucho), es superior a los 4 meses de facturación y en otros casos supera los 6 meses, lo que representa más del $50 \%$ de la facturación anual. Es evidente que si las empresas lograran reducir las pérdidas de agua y redujesen la morosidad ello les permitiría obtener mayores ingresos. Estos dos indicadores son sólo una muestra de las marcadas ineficiencias de gestión de las empresas, que se reflejan también en otros aspectos, por ejemplo en el exceso de trabajadores con relación al número de conexiones. Pero sería errado asumir que la causa de las dificultades financieras se encuentra exclusivamente en las deficiencias de gestión en las empresas; otra causa muy importante es su irracional política tarifaria.

El estudio realizado por ADERASA (2004) evidencia que las tarifas de las empresas de agua potable de Lima, Arequipa y Trujillo se encuentran en el bloque más bajo de la región. Ello se muestra en el Gráfico 1. De otro lado, el Gráfico 2 muestra que las seis empresas seleccionadas cobran una tarifa promedio que resulta insuficiente para cubrir sus costos reales. Esta situación se repite prácticamente en las 54 empresas a nivel nacional. El costo real se ha determinado a partir de los egresos en el Estado de Resultados Financieros y una anualidad del Patrimonio contable, con una tasa de descuento del $11 \%$. La tarifa media se calcula a partir de los ingresos de las empresas divididos por la cantidad $\mathrm{de}^{3}$ facturados.

La situación de tarifas que no cubren los costos reales puede mantenerse por largo tiempo en una industria caracterizada por grandes inversiones en infraestructura, ya que sólo se requiere que la tarifa cu- 


\section{Grafico 1.}

\section{Gasto en servicios de agua y alcantarillado}

para consumo mensual de $20 \mathrm{~m}^{3}$

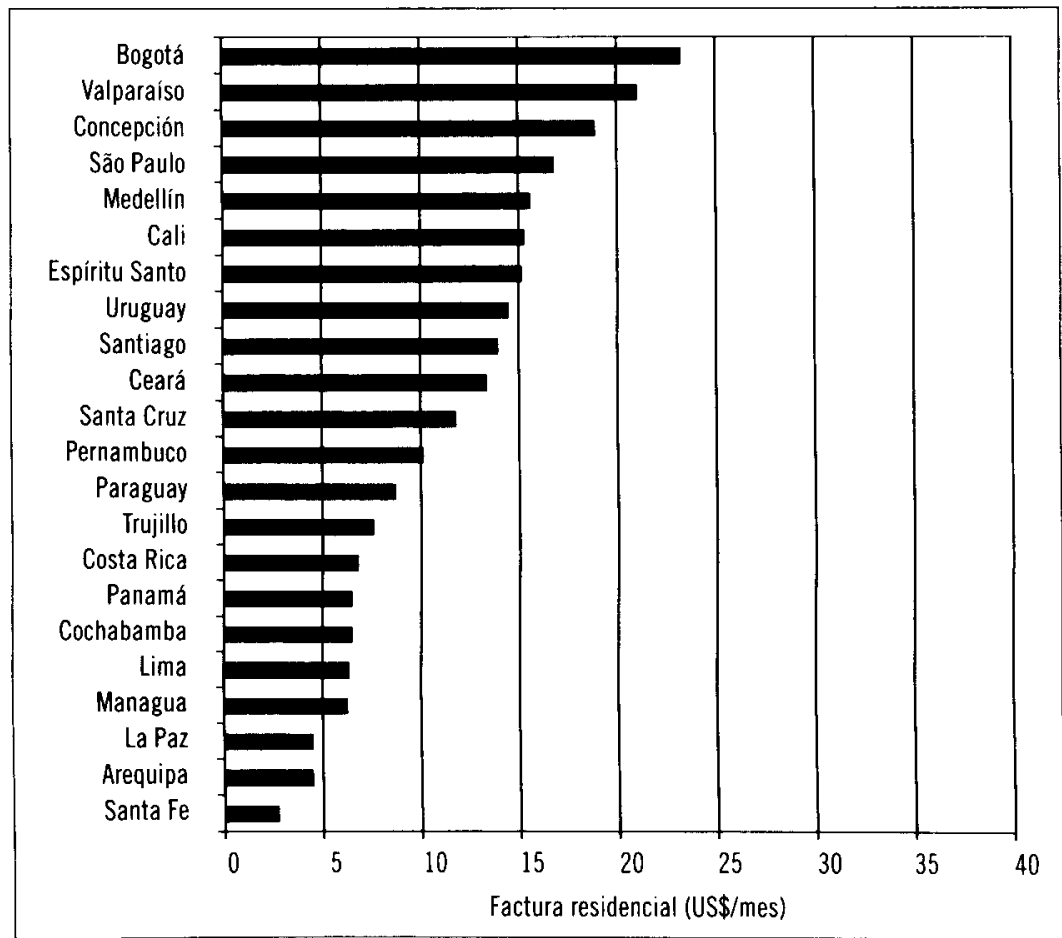

Fuente: ADERASA (2004).

bra los costos de operación y mantenimiento para que el servicio sea viable. Sin embargo, esta situación implica que no se realizarán inversiones de reposición ni nuevas, y que el capital invertido se consumirá en el tiempo. Un gran problema en las empresas de agua es que los ingresos son tan bajos y las ineficiencias de gestión tan grandes que también se ha visto afectado el mantenimiento y operación de la infraestructura y, en consecuencia, la calidad del servicio para los que ya tienen acceso.

De otro lado, esta situación crea un círculo vicioso porque para mejorar la eficiencia de gestión las empresas requieren recursos que las dificultades financieras les impiden tener disponibles 5 . Por ejemplo, para reducir el agua no contabilizada es necesario invertir en medido- 
res domiciliarios y en macromedidores para las redes. Asimismo, para reducir la morosidad se requiere invertir en un mejor control de las conexiones clandestinas.

Las empresas han llegado a esta situación luego de un largo proceso de deterioro, en el cual les corresponde una gran responsabilidad a las autoridades municipales, que no han contribuido a promover una gestión empresarial eficiente, ni a mantener niveles tarifarios adecuados, esencialmente por consideraciones políticas ${ }^{6}$. De otro lado, la normativa sobre el sector saneamiento contiene también algunas normas que conducen a una gestión ineficiente; así por ejemplo, la Ley establece que los directores de las empresas son designados para períodos de un año. Ello ha determinado una rotación demasiado alta de estos directores, lo cual se refleja también en la inestabilidad de los gerentes, quienes suelen ser removidos con demasiada frecuencia. Por su parte, la normativa presupuestal establece severas limitaciones para la gestión eficiente de las empresas.

En adición, el sistema tarifario incluye un sistema de subsidios indiscriminado, que favorece a la gran mayoría de usuarios domésticos. Así, familias de los sectores más pudientes de la sociedad reciben un subsidio importante a los servicios de agua y desagüe sin que para ello exista justificación alguna. El siguiente cuadro muestra los niveles de subsidio a las familias, implícito en las tarifas que cobra SEDAPAL.

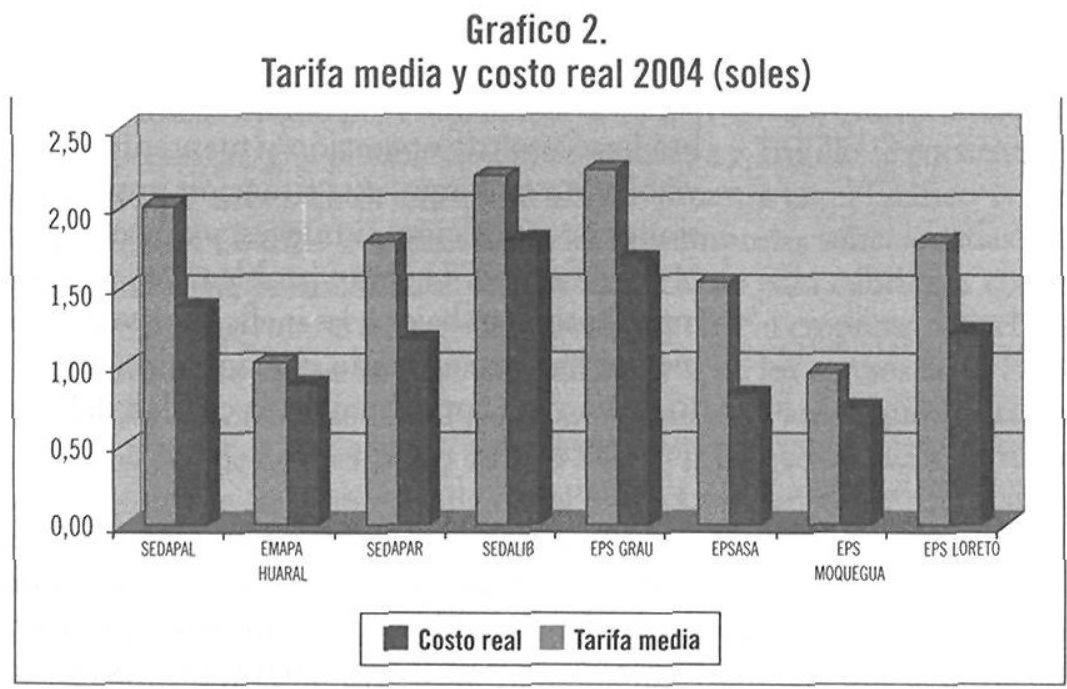




\begin{tabular}{|c|c|c|c|c|c|}
\hline \multirow{2}{*}{$\begin{array}{l}\text { Consumo } \\
\left(\mathrm{m}^{3} / \mathrm{mes}\right)\end{array}$} & \multirow{2}{*}{$\begin{array}{c}\text { Pago } \\
\text { doméstico } \\
\text { (S/. I mes) }\end{array}$} & \multirow{2}{*}{$\begin{array}{c}\text { Tarifa media } \\
\text { doméstica } \\
\left(\mathrm{S} / . / \mathrm{m}^{3}\right)\end{array}$} & \multirow{2}{*}{$\begin{array}{c}\text { Costo } \\
\text { real (*) } \\
\text { (SI. / mes) }\end{array}$} & \multicolumn{2}{|c|}{ Subsidio } \\
\hline & & & & $\begin{array}{l}\text { Costo-Pago } \\
\text { (SI./ mes) }\end{array}$ & $\%$ de costo \\
\hline 20 & 17,88 & 0,89 & 40 & 22,12 & $53,3 \%$ \\
\hline 30 & 29,08 & 0,97 & 60 & 30,92 & $51,5 \%$ \\
\hline 40 & 44,71 & 1,12 & 80 & 35,29 & $44,1 \%$ \\
\hline 50 & 62,08 & 1,24 & 100 & 37,92 & $37,9 \%$ \\
\hline 60 & 83,54 & 1,39 & 120 & 36,46 & $30,4 \%$ \\
\hline
\end{tabular}

* Tarifas vigentes en 2005 .

Se observa que, para un consumo de $30 \mathrm{~m}^{3}$ el subsidio es casi 31 soles $^{7}$, mayor al $50 \%$ del costo real, y aunque porcentualmente el subsidio disminuye con el mayor consumo, el monto en soles tiende a incrementarse. Si bien el subsidio se justifica para las familias más pobres, el sistema actual favorece a todos los consumidores domésticos, independientemente de su condición económica. Esta situación se repite con pequeñas diferencias en las demás ciudades.

Lo dramático del problema es que usualmente los sectores más pudientes reciben un servicio de mejor calidad, usualmente de 24 horas, mientras que los sectores más pobres pueden recibir el agua apenas 2 ó 3 horas por día. Un balance entre el servicio recibido y el monto pagado permitiría apreciar que, debido a la menor calidad del servicio que reciben con relación al monto que pagan, los usuarios más pobres subsidian en la práctica a los más ricos. Más aún, la injusticia de la política de subsidios vigente alcanza niveles extremos en el caso de la denominada "tarifa social». La tarifa social es la que se aplica a familias, usualmente pobres, que reciben servicios en piletas públicas o en viviendas multifamiliares donde existe un solo caño común fuera de la casa ${ }^{8}$. Por tanto, ésta no es realmente una tarifa "social" que se otorgue para favorecer a los más pobres, sino que corresponde a un servicio fuera del domicilio, que objetivamente es de una calidad menor, y que por ello debería tener un precio inferior. Sin embargo, en muchas ciudades esta tarifa social es igual a la tarifa para el primer bloque de consumo de cualquier usuario doméstico. Por ejemplo en Lima, la tarifa social es la misma que se aplica hasta $20 \mathrm{~m}^{3}$ de consumo en cualquier zona de la 
ciudad, incluyendo los distritos donde habitan las familias más pudientes. En este contexto, las familias de ingresos altos, cuyo consumo sea de hasta $20 \mathrm{~m}^{3}$, pagan exactamente lo mismo por $\mathrm{m}^{3}$ que una familia pobre cuyo servicio es de una calidad significativamente inferior. Ello evidencia que el subsidio cruzado va de las familias pobres a las pudientes.

Cabe destacar, por otra parte, que las campañas realizadas en contra de la participación privada en los servicios de saneamiento han mantenido fuera de la agenda el problema de las bajas tarifas promedio y el de los subsidios indiscriminados. Lo cual revela que la corrección de los problemas tarifarios de las empresas resulta poco atractiva políticamente, no obstante que su continuidad afecta severamente a las familias más pobres, que no tienen acceso a los servicios, o que reciben un servicio de muy baja calidad. La corrección del subsidio indiscriminado podría permitir a las empresas obtener mayores ingresos y aliviar su situación financiera, lo que las pondría en mejores condiciones para ampliar el servicio a las familias más necesitadas. Ciertamente, se requiere también mejorar la gestión de las empresas, o de lo contrario los mayores ingresos podrían ser dilapidados en mayores ineficiencias, pero es claro que pretender mejorar la gestión sin elevar la tarifa promedio sería una respuesta incompleta.

\section{LOS REQUERIMIENTOS DE INVERSIÓN Y LA PARTICIPACIÓN PRIVADA}

Uno de los principales problemas para el desarrollo de este sector ha sido la insuficiente inversión realizada en los últimos años, en contraste con los elevados requerimientos para alcanzar las Metas del Milenio: reducir a la mitad las personas que carecen de acceso a los servicios de agua y alcantarillado.

El siguiente cuadro muestra los elevados requerimientos de inversión en el sector, lo cual evidencia que difícilmente el Estado dispondría de recursos suficientes para alcanzar las metas planteadas. En este contexto, algunos plantean que la participación de inversionistas privados podría aportar los recursos necesarios, además que permitiría superar las severas deficiencias de gestión que caracterizan a las empresas municipales. 


\begin{tabular}{|l|c|c|c|}
\hline \multirow{2}{*}{ Servicio } & \multicolumn{2}{|c|}{ Cobertura nacional } & \multirow{2}{*}{$\begin{array}{c}\text { Gráfico 2 } \\
\text { (US\$ Millones) }\end{array}$} \\
\cline { 2 - 3 } & $\mathbf{2 0 0 3}$ & $\mathbf{2 0 1 5}$ & 1.479 \\
\hline Agua potable & $\mathbf{7 5 \%}$ & $82 \%$ & 1.350 \\
\hline Saneamiento & $57 \%$ & $77 \%$ & 1.643 \\
\hline Tratamiento $^{1}$ & $16 \%$ & $97 \%$ & $\mathbf{4 . 4 7 2}$ \\
\hline \multicolumn{3}{|c|}{ TOTAL } \\
\hline
\end{tabular}

Porcentaje estimado sobre la población urbana servida con sistemas de alcantarillado.

Fuente: Presentación del Viceministro de Construcción y Saneamiento, Jorge Villacorta, en diciembre de 2004.

La posibilidad de una gestión privada de los servicios ha generado mucha controversia e incluso se ha formado un activo movimiento de numerosas ONG opuesto a esta opción. Para este sector de opinión, el reconocimiento de un derecho humano al agua potable implicaría reconocerla como un bien público y rechazar su valor económico. En efecto, Alternativa y Cedal (2004) plantean que el agua es un recurso natural limitado y en consecuencia un bien público para la vida y la salud. El concepto de bien público, en este caso, se refiere a que el agua debería ser un bien de dominio público, por lo cual debería rechazarse el establecimiento de derechos de propiedad privados sobre la misma. En economía un bien público no es lo mismo que un bien de dominio público. Desde el punto de vista económico, un bien público tiene dos características principales: a) Cuando está disponible para un consumidor está también disponible para los demás consumidores, es decir, no se puede excluir a nadie del consumo de dicho bien; b) El consumo de un bien público por un consumidor no disminuye el consumo de los demás consumidores, es decir, no existe rivalidad en el consumo de un bien público. Una característica del agua es que tiene las propiedades de un bien público cuando se encuentra en sus fuentes naturales. Sin embargo, una vez que empieza a ser utilizada en la agricultura o el servicio de agua potable asume las características de un bien privado. Aun cuando usualmente el agua de riego se maneja en forma asociativa, y las aguas utilizadas por un agricultor pueden ser utilizadas más abajo por otros agricultores, en muchos casos el consumo de agua por un agricultor puede impedir su uso por otros agricultores. Por tanto, aunque no de manera absoluta, existe rivalidad en 
el consumo. Alguien podría construir un canal para captar agua de riego, siempre que contara con las autorizaciones respectivas, y podría excluir a los demás del uso de este recurso. En cuanto al servicio de agua potable, éste claramente puede excluir a quienes no paguen por el servicio. Cabe señalar, de otro lado, que el uso de las aguas como receptor de residuos de diversa naturaleza corresponde también a las características de un bien (mal) público.

En consecuencia, desde un punto de vista puramente económico, el agua es a la vez un bien privado y un bien público ${ }^{9}$ y ello justifica que la asignación del recurso sea efectuada con la intervención del Estado, en base a criterios económicos, pero para lograr objetivos sociales. Se entiende entonces que el reconocimiento del valor económico del agua no impide realizar su gestión con criterios de interés público, ni tampoco que la legislación pueda considerarla como un bien de dominio público. En el caso de los servicios de agua potable y alcantarillado, el acceso universal genera externalidades sanitarias positivas en tanto reduce la posibilidad de epidemias de enfermedades gastroentéricas, además de que el solo hecho de saber que existe un acceso universal genera bienestar para la sociedad, mientras que la existencia de familias sin acceso genera malestar o pérdida de bienestar económico ${ }^{10}$. Sin embargo, el reconocimiento del acceso al agua potable y alcantarillado como un derecho humano, que obliga al Estado a garantizar el acceso universal a estos servicios, no implica necesariamente que deban manejarse como bienes de dominio público. El caso de Chile evidencia que es posible garantizar el derecho al agua mediante un sistema adecuado de subsidios, aun si el Estado privatiza dichos servicios. En ese país las empresas de agua potable y alcantarillado se han privatizado, e incluso se aplican derechos de propiedad sobre el agua, y sin embargo el Estado otorga un subsidio a las familias pobres para un consumo mensual gratuito de hasta $20 \mathrm{~m}^{3}$ de agua potable. De otro lado, no es indispensable establecer la vigencia de derechos de propiedad sobre el agua para que exista una gestión privada de los servicios de agua potable y alcantarillado, como se observa en varios países de América Latina que han privatizado sus servicios.

Por tanto, el reconocimiento del derecho humano al agua no tiene por qué contraponerse con el reconocimiento de su valor económico, ni tampoco con la posibilidad de una gestión privada de los servicios 
de agua potable y alcantarillado. Siguiendo a Perry et al., las decisiones sobre el grado en que se permite la participación privada en la gestión del agua deberían responder a las circunstancias de cada caso particular. Es decir, que deberían sustentarse en consideraciones prácticas, antes que ideológicas. Bajo este marco, difícilmente puede sostenerse que la privatización es la panacea que puede resolver todos los problemas de los servicios de agua potable en el Perú. Por un lado, Estache y Rossi (2002), en un estudio para 50 empresas en 29 países de la región Asia-Pacífico, encontraron que la eficiencia de las empresas privadas de agua no era significativamente diferente de las públicas. Asimismo, Clarke, Kosec y Wallsten (2004), en base a encuestas de hogares domésticos en Argentina, Bolivia y Brasil, afirman que existe poca evidencia de que la privatización por sí misma mejore el acceso a los servicios de agua. Ellos señalan también que la privatización no parece haber sido negativa para los pobres.

De otro lado, en muchas ciudades pequeñas del Perú se carecería de los incentivos suficientes para atraer inversionistas privados, por lo cual en tales casos no existiría otra opción que la gestión pública del agua potable. Pero, del mismo modo, difícilmente se podría negar que la gestión pública de estos servicios haya fracasado en el Perú, no obstante los intentos de reforma que se han ejecutado, incluyendo la inyección de cuantiosos recursos de la cooperación técnica y financiera. Es indudable, por tanto, que existe un espacio para la participación de inversionistas privados, en la forma de concesiones $u$ otras formas de participación privada en los servicios de agua que, bajo condiciones adecuadas, podría contribuir a ampliar la cobertura y mejorar la calidad de estos servicios.

Sin embargo, la presencia de riesgos importantes en el otorgamiento de concesiones privadas para la prestación de los servicios de agua potable y alcantarillado, aunque no justificaría dejar de lado esta opción, sí aconseja mucha cautela ${ }^{11}$. En primer lugar, la onerosa condicionalidad que caracteriza a los contratos de concesión privada crea la posibilidad de que el Estado, en caso que fracase la privatización, se vea enfrentado a importantes pasivos como consecuencia de las demandas de las empresas ante los centros internacionales de arbitraje. En los casos de Bolivia y Argentina, el fracaso de la privatización de los servicios de agua ha generado demandas multimillonarias a los Estados de dichos países, con una alta posibilidad de éxito para las empresas demandantes. 
En segundo lugar, los países donde se enfrentan problemas de debilidad institucional del Estado suelen tener grandes dificultades para establecer organismos reguladores técnica y políticamente fuertes, capaces de realizar una regulación eficiente de estos servicios. La recomendación usual es que los organismos reguladores se establezcan con suficiente anticipación a los procesos de concesión, de tal manera que puedan entrenarse con la regulación de las empresas mientras éstas son públicas. No obstante que en Perú tal criterio se ha cumplido con el organismo regulador SUNASS, éste ha cambiado tres gerentes generales en los últimos cuatro años, lo cual inevitablemente debilita su capacidad institucional.

Un tercer riesgo importante proviene de la vulnerabilidad al ataque político de las privatizaciones de los servicios de agua. No es casual que los principales fracasos de los procesos de privatización en América Latina se hayan producido en el caso del agua potable y alcantarillado, en Bolivia y Argentina. Un factor que puede contribuir al fracaso de la privatización son las expectativas insatisfechas, generalmente sobredimensionadas. Por ello, es muy importante que la privatización se realice luego de amplios procesos de debate público, en los cuales se proporcione amplia información a la población sobre las condiciones planteadas, de tal manera que pueda garantizarse un apoyo claro y mayoritario al otorgamiento de la concesión privada.

Como puede apreciarse, ni la privatización ni la continuidad de la gestión pública constituyen soluciones generales a los problemas de desarrollo de los servicios de agua potable y alcantarillado en el Perú, pero una combinación inteligente de ambas, en la que se opte por concesiones privadas en ciertos casos, asociaciones público-privadas en otros y gestión pública en los demás, podría ofrecer mejores resultados, acordes con la realidad de cada caso particular. Cabe destacar, sin embargo, que aun en ciudades donde la participación privada podría ser viable económicamente, la fuerte oposición de sectores organizados de la población al otorgamiento de concesiones privadas podría hacerlas inviables políticamente.

En tal sentido, la existencia de distintas opciones para la participación privada permitiría adaptarla a las circunstancias locales. En los casos de Tumbes y Piura se ha optado por la concesión privada, pero en el caso de SEDAPAL en Lima se tiene un contrato BOT (Construir, 
Operar y Transferir) por el cual un consorcio privado ha invertido en la construcción de una planta de tratamiento de agua potable en el río Chillón, la cual operará durante 30 años, luego de lo cual será transferida a SEDAPAL. También existen los denominados Contratos de Gerencia, en los cuales un privado se compromete a administrar una empresa por un período de 5 a 10 años, luego de lo cual se podría pasar a una concesión privada o el servicio podría retornar a ser administrado por la empresa municipal. Estas últimas opciones pueden ser aceptables para los sectores que reconocen la gravedad de los problemas de las empresas de agua, pero que rechazan las concesiones privadas.

Es importante destacar, por otra parte, que en aquellas empresas en las que no haya condiciones para una participación privada, resulta indispensable que el Estado disponga de recursos públicos para efectuar las inversiones necesarias, así como medidas urgentes que permitan mejorar la gestión empresarial y corregir las distorsionadas estructuras tarifarias. Esto implica llevar a cabo una reforma radical del marco institucional para la gestión pública de las empresas de agua, que haga posible liberarlas del control municipal, y que otorgue una adecuada autonomía a los gerentes para que sus decisiones sean tomadas estrictamente bajo consideraciones empresariales y de interés público.

\section{CONCLUSIÓN}

El acceso a servicios adecuados de agua y desagüe es un derecho humano reconocido por pactos internacionales de los cuales el Perú es parte, y además este derecho se desprende de derechos reconocidos por la Constitución, como el derecho a la vida y el derecho a la salud. De otro lado, el reconocimiento de un valor económico en el agua no se contrapone con el derecho humano al agua, ni tampoco implica que su asignación deba realizarse a través del libre juego de las fuerzas del mercado, ya que existen externalidades y consideraciones sociales que hacen necesaria una regulación por parte del Estado. Asimismo, tampoco existe contraposición entre el reconocimiento del derecho al agua y la participación de inversionistas privados en la prestación de los servicios de agua potable y alcantarillado.

La grave situación financiera en que se encuentran la mayoría de empresas municipales prestadoras de estos servicios es el principal 
obstáculo para que no puedan garantizar el derecho al agua a las poblaciones dentro de su ámbito de operaciones. Tal situación sólo podrá superarse en la medida que se corrijan las dos causas que originan el problema: la ineficiente gestión de las empresas y los bajos niveles tarifarios. Esto último puede resolverse parcialmente mediante la eliminación del actual sistema de subsidios indiscriminados, para que sólo favorezcan a quienes realmente los necesitan. Pero además, el sistema de subsidios actual no sólo es parte del problema financiero de las empresas, sino que además configura una situación de desigualdad e injusticia mayúscula que desfavorece a los sectores de menores ingresos en la sociedad.

Finalmente, los elevados montos de inversión necesarios en el sector agua y saneamiento hacen indispensable la convocatoria a la inversión privada en sus diversas formas, de manera complementaria con la inversión pública. Por tanto, antes que hacer una oposición ideológica a la gestión privada de los servicios, sería aconsejable establecer los términos de una participación privada que sea adecuada a la realidad de cada zona, de tal manera que se pueda contar con recursos importantes para financiar las inversiones necesarias, y así garantizar la vigencia del derecho humano al agua. Asimismo, el Estado no debe desentenderse de su responsabilidad de regular la prestación de los servicios de agua para garantizar este derecho a todas las personas, por lo que debería adoptar las medidas necesarias para una gestión eficiente de las empresas estatales, aportar los recursos de inversión necesarios allí donde no sea posible o aconsejable conseguir recursos privados, y también mantener un sistema de subsidios orientado exclusivamente a los más pobres.

\section{NOTAS}

1. Defensoría del Pueblo de Perú (2005).

2. Fondo Nacional de Vivienda, creado con aportes de los trabajadores para financiar el acceso a la vivienda, $y$ utilizado extensivamente por el gobierno del ex presidente Fujimori para financiar obras de agua, saneamiento y electricidad.

3. Sin embargo, tanto el gobierno central, como los gobiernos regionales y locales suelen realizar directamente algunas obras, aunque insuficientes para las necesidades existentes.

4. Un serio problema de la morosidad es que las empresas deben pagar a la SUNAT el IGV por las facturas no cobradas. Debido a ello algunas empresas han 
recurrido a no emitir facturas a los morosos que se reconectan clandestinamente, a fin de evitar la deuda con la SUNAT. Esto podría explicar por qué SEDACUSCO y EPSASA muestran un indicador de morosidad bajo.

5. A esto también se le suele denominar una trampa de bajo nivel.

6. En el año 2005 la SUNASS aprobó un programa de incrementos tarifarios para SEDAPAR (Arequipa) que no fue aplicado por decisión de las autoridades municipales.

7. Tipo de cambio 3,3 soles por dólar.

8. También se aplica a otros usuarios como conventos y casas de asilo.

9. Perry et al. (1997), Gleick et al. (2002) y Hanemann (2005).

10. Las personas en general están dispuestas a pagar más por el agua potable con la finalidad de subsidiar a quienes no pueden pagar por ésta.

11. Otros riesgos de la privatización de los servicios de agua y la manera de enfrentarlos se analizan en Gleick et al. (2002).

\section{BIBLIOGRAFIA}

ADERASA: Las tarifas de agua potable y alcantarillado en América Latina. Grupo de Tarifas y Subsidios, 2005. http://www.aderasa.org/es/documentos3.htm? $\mathrm{x}=654$

Alternativa y Cedal: Desafios del Derecho Humano al Agua en el Perú, Lima, Perú 2004.

Briscoe: "Water as an economic good: the idea and what it means in practice", The World Bank, a paper presented at the World Congress of the International Commission on Irrigation and Drainage, Cairo 2004.

Clarke, G. R. G.; Kosec, K. y Wallsten, S.: Has private participation in water and sewerage improved coverage? Empirical evidence from Latin America, World Bank Policy Research Working Paper, 3445, November 2004.

Defensoría del Pueblo: Ciudadanos sin Agua: Análisis de un Derecho Vulnerado, Informe Defensorial N. 94 , Lima, Perú 2005.

Defensoría del Pueblo de Colombia: El Derecho Humano al Agua en la Constitución, la Jurisprudencia y los Instrumentos Internacionales, Bogotá 2005.

Estuche, A. y Rossi, M.: How different is efficiency of public and private water companies in Asia? The World Bank Economic Review, Vol 16. (1), pp. 139-148, Washington D.C. 2002.

Gleick, P. H: "The human right to water" en Water Policy 1 (1998), 487-503. Gleick, P. H.; Wolff G.; Chalecki, E. L. y Reyes R.: "The new economy of water: the risks and benefits of globalization and privatization of fresh water", Pacific Institute for Studies in Development, Environment and Security, California, 2002.

Hanemann, M.: The value of water, University of California Berkeley, 2005. http://are.berkeley.edu/courses/EEP162/spring05/valuewater.pdf 
Perry, C. J.; Rock, M. y Seckler, D.: Water as an economic good: a solution or a problem? Research Report N. ${ }^{\circ} 14$, International Irrigation Management Institute, Sri Lanka.1997.

Scanlon, J.; Cassar, A. y Nemes, N.: Water as a human right? IUCN Environmental Policy and Law Paper N.o 51, International Union for Conservation of Nature and Natural Resources, UK. 2004.

\section{ABSTRACT}

Based on international agreements and the Peruvian Constitution this paper argues that the legal framework in Perú recognizes a right to water supply. The causes why this right is actually not in force are analyzed, particularly with regard to the managerial and economic constraints faced by the state own companies. Also, the concepts of public good and public domain good are discussed as a framework to analyze the alleged conflict between the recognition of a human right to water and the economic value of this resource. The author makes a case for private involvement to provide management capabilities and capital based on the lack of both in the public sector. However, the risks of private intervention are also analyzed and this is considered complementary rather than opposite to the public management.

Key words: human rights, water supply, utilities, public management, privatisation. 